\title{
Estudio de Diseño de Diagramas de Operación en Sistemas de Supervisión, Control \& Adquisición de Datos en Tiempo Real "SCADA" para Subestaciones de Distribución
}

Ing. Luis Panchi Alvear e Ing. (e) Freddy S. Iza G.

lpanchi@eeq.com.ec

\section{Resumen}

Este artículo presenta el resultado de un estudio para determinar un procedimiento lógico de diseño de diagramas de operación en sistemas de supervisión, Control \& Adquisición de Datos en Tiempo Real (SCADA), orientado a la operación de redes eléctricas de distribución, con lo cual se busca estandarizar la utilización de los diferentes esquemas gráficos empleados en la operación del Sistema Eléctrico Quito (SEQ).

Como parte del procedimiento científico se ubican físicamente los diferentes dispositivos (disyuntores) tanto en cabinas de operación como en los patios de maniobras, con lo cual se identifica que en las subestaciones de distribución, en las cuales se tiene un nivel de tensión de $6,3 \mathrm{kV}$, los dispositivos se encuentran distribuidos en cabinas; en las subestaciones de distribución de un mayor nivel de tensión $(23 \mathrm{kV})$, los dispositivos se encuentran en el patio de maniobras y para las subestaciones que tienen los dispositivos con aislamiento en SF6, su distribución es en cabinas.

Palabras clave: Sistema de Control Supervisorio \& Adquisición de Datos (SCADA), Interfaz Humano Máquina (IHM), Interfase Gráfica de Usuario (GUI), Interfase Hombre Máquina (MMI), Dispositivo Electrónico Inteligente (IED).
Abstract

This article presents the results of a study to determine a logical design of operating diagrams in Supervisory Control \& Data Acquisition in Real Time (SCADA), oriented to the operation of electrical distribution networks, thereby aims to standardize the use of different graphic schemes used in the operation of Quito Electricity System (SEQ).

As part of the scientific process, are located physically different devices (circuit breakers) operating both in cabins and in the switching yard, which is identified in distribution substations which has a voltage level of $6.3 \mathrm{kV}$, the devices are distributed in boxes, distribution substations of a higher voltage $(23 \mathrm{kV})$, the devices are in the switching yard and substation devices with SF6insulated, their distribution is in cabins.

Keywords: Supervisory Control And Data Acquisition System (SCADA), Human Machine Interface (HMI), Graphical User Interface (GUI), Man Machine Interface (MMI), Intelligent Electronic Device (IED)

Forma sugerida de citar: Panchi Alvear, Luis e Iza G., Freddy. 2011. "Estudio de Diseño de Diagramas de Operación en Sistemas de Supervisión, Control \& Adquisición de Datos en Tiempo Real "SCADA" para Subestaciones de Distribución”. Ingenius No. 5. Enero/Junio. Pp. 3-14. 


\section{Introducción}

Los crecientes avances de la Electrónica y las Tecnologías de la información (TIC ‘s) han determinado cambios muy importantes en los sistemas de automatización, por lo tanto la operación de los diferentes dispositivos de las subestaciones no ha sido indiferente a estos cambios [1].

La Empresa Eléctrica Quito S.A. se encuentra trabajando en la etapa final de un ambicioso proyecto de automatización de su sistema eléctrico, motivada por el mejoramiento del servicio hacia sus clientes y con el fin de aprovechar oportunidades derivadas de tendencias tecnológicas en automatización de subestaciones, SCADA, Comunicaciones, automatización de redes de distribución y en Centros de Control.

Este estudio presenta, en su primera parte, el análisis de la problemática relacionada a la operación de los sistemas actuales de la EEQ S.A. y, en una segunda parte, presenta un plan de acción para el desarrollo de una Interfaz Gráfica de Usuario (GUI) que permite un desarrollo lógico de los diferentes sinópticos relacionados con la operación en tiempo real.

Las conclusiones y recomendaciones constituyen un plan de acción aplicable a empresas eléctricas de distribución que permite identificar que la aplicación de un procedimiento lógico en el diseño de diagramas de operación (sinópticos), es un mecanismo útil.

\section{Componentes}

\subsection{Situación actual de la EEQ S.A.}

La EEQ S.A. es una empresa que tiene su giro de negocio en el sector residencial, comer- cial e industrial de Quito, capital de Ecuador; actualmente se encuentra bajo la supervisión del Centro Nacional de Control de Energía (CENACE), quien a su vez también actúa como ente regulador con el fin de viabilizar la operación técnico/económico del Sistema $\mathrm{Na}$ cional Interconectado (SNI) dentro del sector eléctrico ecuatoriano.

Las subestaciones más importantes por su magnitud son: Santa Rosa, Pomasqui, Selva Alegre y Vicentina. A excepción de la s/e Selva Alegre las demás tienen interconexión con el Sistema Nacional Interconectado (SNI). Las subestaciones de seccionamiento en $46 \mathrm{kV}$ más importantes son: Norte, Sur, Epiclachima, San Rafael y Guangopolo Térmica.

La operación y supervisión del Sistema Eléctrico se realiza a través de dos áreas de despacho, uno de Distribución y otro de Potencia. Despacho de Distribución realiza la supervisión y operación del sistema primario y secundario de distribución. Despacho de Potencia supervisa y controla la operación del sistema a nivel de subtransmisión $46 \mathrm{kV}$ y $138 \mathrm{kV}$, incluyendo la programación de la generación propia.

\subsection{Sistemas de apoyo a la operación}

Actualmente, la EEQ S.A. cuenta con los siguientes sistemas de apoyo para la operación de la red de distribución:

- SCADA SHERPA: Sistema de Control Supervisorio y Adquisición de Datos de 25 subestaciones de distribución-subtransmisión que permite operarlas en forma desatendida.

- GIS: Sistema de Información Geográfica sobre base cartográfica con aplicaciones basados en AutoCAD. Este sistema cuenta con 
información de todas las instalaciones y de conexión cliente-transformador. El sistema corre sobre plataforma Windows XP Profesional con motor de base de datos Oracle 9i y estaciones de operación basadas en PC.

- TCA: Sistema para la atención de llamadas y despacho de cuadrillas. Se encuentra sobre un servidor con sistema operativo UNIX.

- SIDECOM: Sistema de Información Comercial.

- FEDER-ALL: Sistema de Medición de Energía.

- SDI: Sistema de Información de Distribución, que maneja las incidencias del sistema y trabajos programados.

\subsection{Diagnóstico de los sistemas actuales}

La EEQ S.A. está en proceso de integración operativa, con procesos y relaciones propias. En consecuencia se visualiza una evolución hacia una operación más integrada. En relación con los sistemas de apoyo de la operación se ha identificado lo siguiente:

1. Seguridad de la operación. Se requiere mejorar las condiciones de seguridad operativa de manera que se evite cualquier posible inadecuada operación de equipos, por ejemplo, bajo consignación local, es decir cada sistema debe poseer su propio sistema de seguridad [6].

2. Desempeño del sistema. Los sistemas actuales de operación se encuentran al límite de los tiempos de respuesta prácticos que pudieran ser satisfactorios para una operación desatendida de las subestaciones.

\section{Métodos}

\subsection{Esquemas de operación}

En el Sistema Eléctrico Quito (SEQ), se ha utilizado los diagramas unifilares que son desarrollados por el área de Despacho de Potencia en AutoCAD; un ejemplo de este esquema se muestra en la Figura 1.

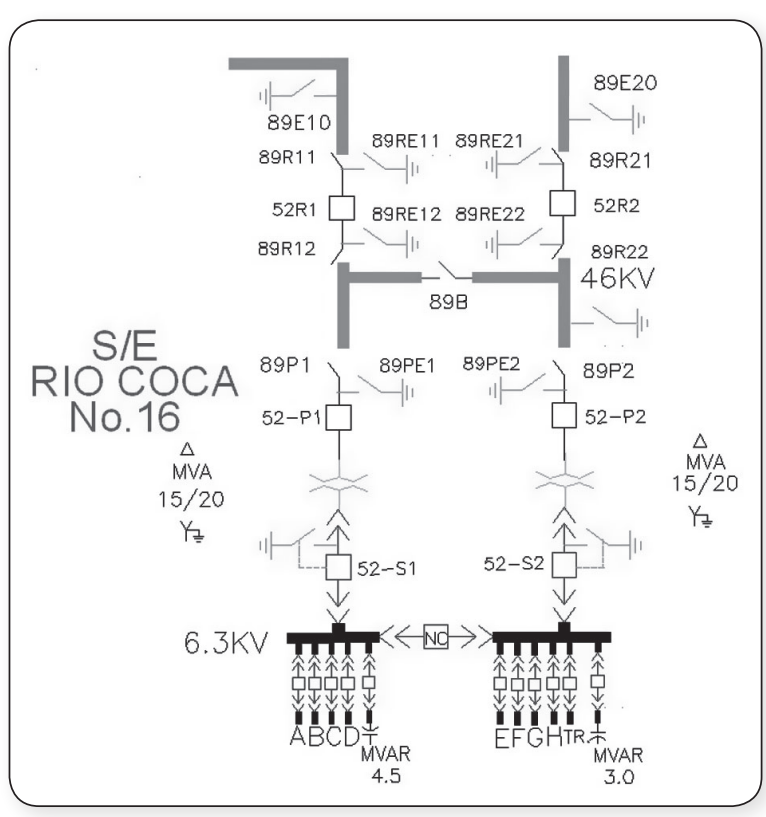

Figura 1. Diagrama unifilar de una subestación

Estos diagramas permiten al personal de operación del Centro de Control tener una perspectiva del sistema eléctrico para su maniobra en condiciones normales o en contingencia.

En la operación de las subestaciones de distribución se ha logrado identificar que los esquemas unifilares, no concuerdan con el orden, ni la ubicación real de los equipos existentes en cada una de las subestaciones. 


\subsection{Filosofía de la operación}

Con la consecución del Proyecto Nuevo Sistema SCADA, se adoptó una filosofia de implementación, en la cual se indican los siguientes criterios:

- Subestaciones de Distribución que tienen reles electromecánicos y tienen un nivel de tensión de $6,3 \mathrm{kV}$, se les denominará como subestaciones de tipo BÁSICO.

- Subestaciones de Distribución que tienen reles electromecánicos e IED's y tienen un nivel de tensión de $23 \mathrm{kV}$, se les denominará como subestaciones de tipo INTERMEDIO.

- Subestaciones de Distribución que tienen IED's y tienen un nivel de tensión de $23 \mathrm{kV}$, se les denominará como subestaciones de tipo COMPLETO.
Esta filosofia se definió de tal forma que con la sustitución de los reles electrónicos y electromecánicos por IED's en las distintas subestaciones, éstas crezcan a un nivel mayor de automatización de la operación, esta filosofia de implementación se muestra en la Figura 2.

Para las subestaciones de tipo COMPLETO, se tiene un Terminal de Operación Local en cada subestación, que con un software de control de la subestaciones, permite realizar la gestión local de las mismas independientemente del Centro de Control Principal, de tal forma que si por algún problema de comunicaciones, o mantenimiento del sistema SCADA del Centro de Control Principal no se pudiera operar, se lo realizaría desde el Centro de Control Local.

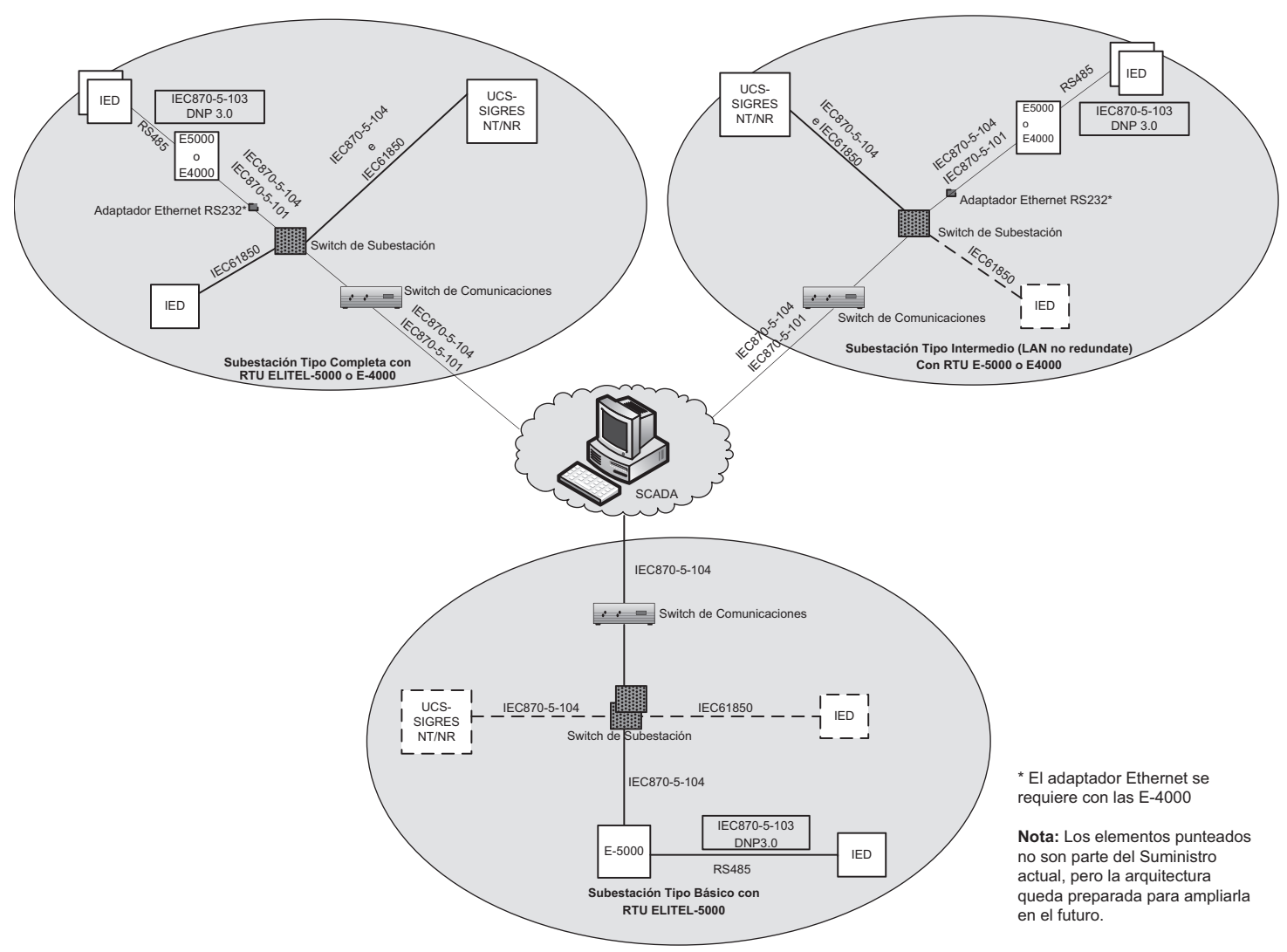

Figura 2. Esquema general de los sistemas propuestos 
Como requerimiento de la EEQ S.A. para nuevas subestaciones comprendidas en su plan de ampliación, estas nuevas subestaciones deben tener su propio SCADA LOCAL, Micro SCADA o Terminal de Operación Local, que le permita realizar cualquier operación de manera desatendida [3].

\section{Enfoque general de las soluciones propuestas}

\subsection{Objetivos}

Luego del análisis de la situación actual, se identificaron dos objetivos estratégicos para el desarrollo del estudio:

- Mejorar la operación del usuario del sistema SCADA, de manera que fácilmente pueda identificar la configuración y el orden de los dispositivos, dependiendo del tipo de subestación.

- Lograr la mejor eficiencia y eficacia en la operación del sistema eléctrico.

En cumplimiento de los dos objetivos estratégicos, se pueden fijar los siguientes objetivos específicos:

- Cumplir los índices FMIK (Frecuencia Media de Interrupción del Servicio por kVA instalado) y TTIK (Tiempo Total de interrupción de Servicio por kVA instalado) requeridas por la regulación vigente, Como se muestran en (1) y (2):

- Reducir el tiempo de atención de daños, con miras a disminuir los tiempos de detección y aislamiento de fallas y de restauración del servicio.

$$
F M I K_{R d}=\frac{\sum_{i} k V A f s_{i}}{k V A_{\text {inst }}}
$$

$\operatorname{TTIK}_{R d}=\frac{\sum_{i} k V A f s_{i} * T f s_{i}}{k V A_{\text {inst }}}$

\subsection{Soluciones propuestas}

Con base en el análisis del estudio realizado se propone implantar soluciones que respondan tanto a los aspectos de cumplimiento de la regulación en lo que respecta a la calidad de servicio como los demás requisitos regulatorios y de operación mediante la implementación de las siguientes medidas:

- Adopción de un estándar de señalización, medida y mando remotos: Este estándar sería la base para llevar la información necesaria de todas las subestaciones.

Esta adopción permitirá uniformizar la información de los diagramas de operación y de inventario, ya que basándose en la actualización de los diagramas de operación, se actualizarán también los diagramas unifilares de las subestaciones.

\subsection{Esquemas de operación en tiempo real}

El interfaz hombre-máquina (IHM) que posee el SCADA nos muestra el diagrama unifilar de las subestaciones (sinóptico), con los estados de los elementos de interrupción (disyuntores, seccionadores), valores de medidas analógicas tales como voltajes, corrientes y potencias y el estado de las comunicaciones con los dispositivos electrónicos de la subestación como se muestra en la Figura 3. 


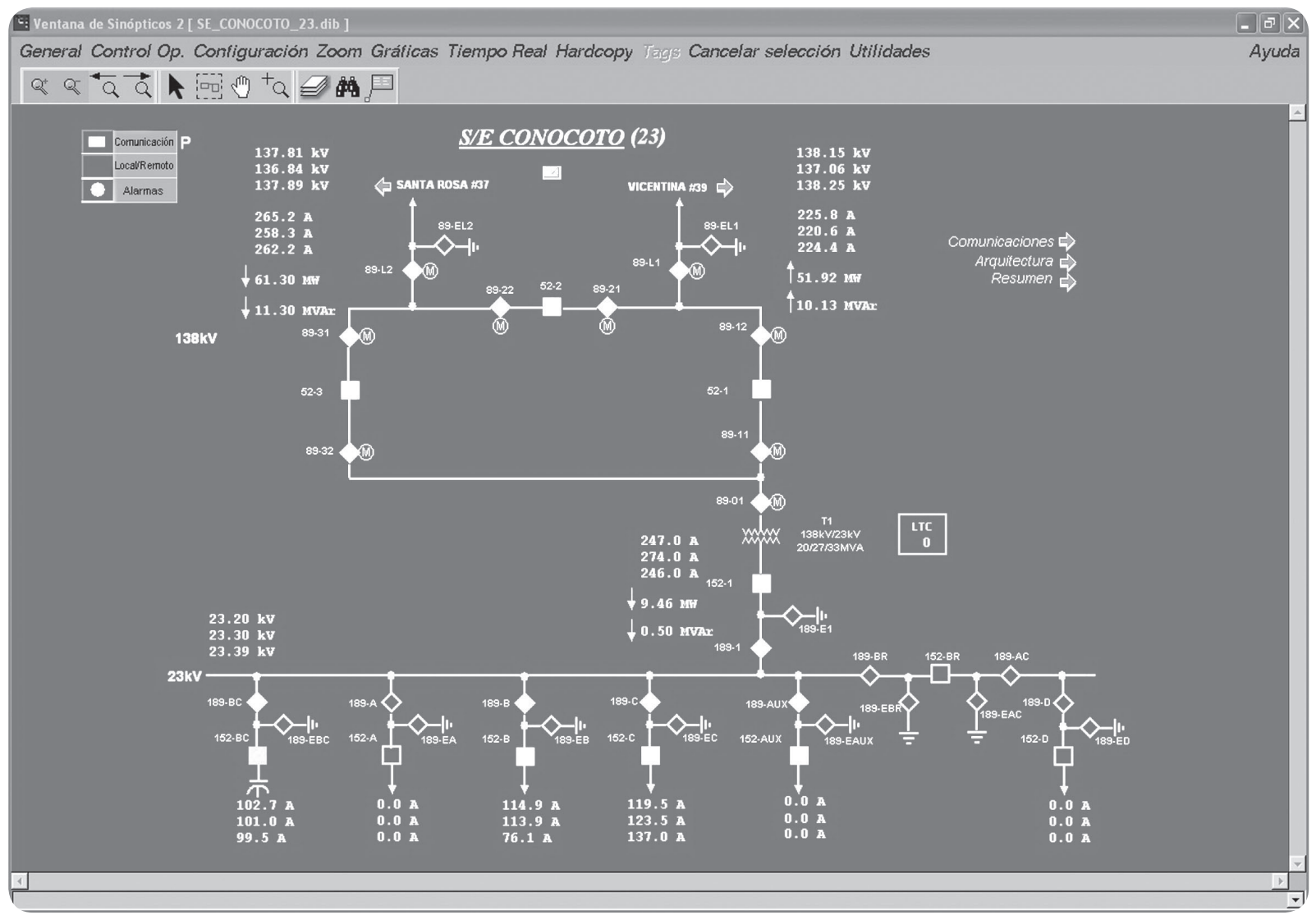

Figura 3. Sinóptico tiempo real de la S/E Conocoto

\subsection{Operación del sistema eléctrico}

En el proceso de automatización de las subestaciones, éstas quedan desatendidas, de modo que los diagramas unifilares de operación deben contener información precisa de la ubicación de los disyuntores, sea que se encuentren en cabinas como es el caso de los disyuntores de 6,3 $\mathrm{kV}$ o en el patio de maniobras como los disyuntores de $23 \mathrm{kV}$, utilizados en el sistema de distribución de la EEQ S.A.

Esta información será proporcionada desde el Centro de Control al personal que tenga que operar en sitio los elementos de la subestación.

\section{Información gráfica para la operación de las subestaciones}

\subsection{Subestaciones básicas de 6,3 kV}

La arquitectura de una subestación básica consta de una RTU que sube al SCADA las señales de los estados de los disyuntores y seccionadores, medidas de voltajes y corrientes y estados de protecciones de los relés electromecánicos en protocolos de comunicación IEC 60870-5-101, 103 y DNP3.

Para nuestro estudio tomaremos como ejemplo a la S/E Diez Vieja, cuyo diagrama 
unifilar se muestra en la Figura 4. El voltaje nominal de distribución es de $6,3 \mathrm{kV}$, cuya barra consta de:

- Un seccionador de barra: 152-SB

- Cinco primarios: 152-A, 152-B, 152-C, 152D, 152-E

- Un Banco de capacitores de 3 MVAR: 152$\mathrm{BC}$

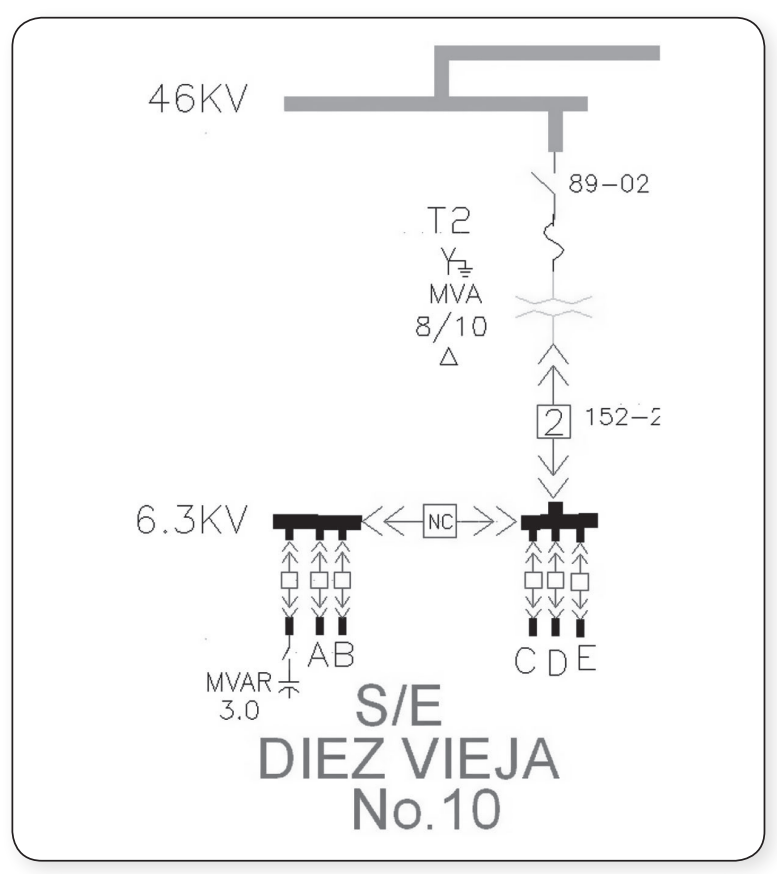

Figura 4. Diagrama unifilar de la S/E Diez Vieja

Físicamente las cabinas de los disyuntores se encuentran ubicados en el siguiente orden de izquierda a derecha: E, D, C, B, A y Banco de capacitores, como se indica en la Figura 5.

Para nuestro sistema SCADA, el sinóptico debe conservar este orden para su operación, como se indica en la Figura 6.

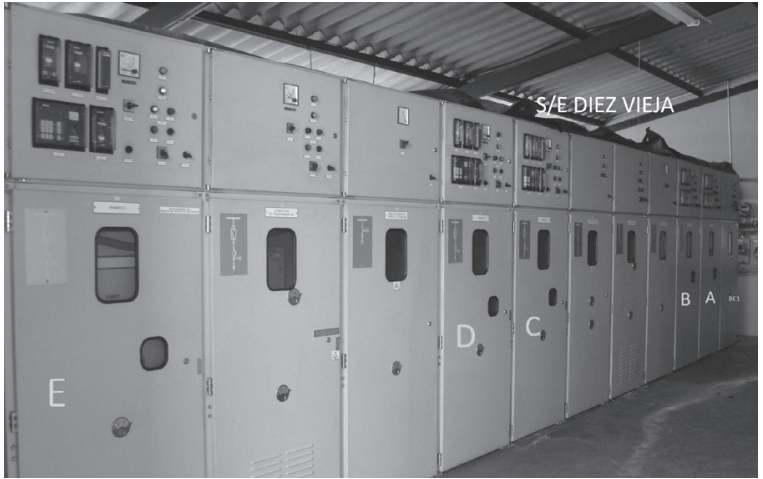

Figura 5. Posición de las cabinas de 6,3 kV en la S/E Diez Vieja

\subsection{Subestaciones intermedias y completas de $23 \mathrm{kV}$}

La arquitectura de una subestación intermedia y completa consta de una RTU, Gateway e IEDs que suben al SCADA las señales de los estados de los disyuntores y seccionadores, medidas de voltajes y corrientes y estados de protecciones en protocolos de comunicación IEC 61850 [5].

La diferencia entre una subestación intermedia y una completa radica principalmente en el Terminal de Operación Local. Las subestaciones completas poseen dicho Terminal con un interfaz HMI, el cual contiene los sinópticos de la subestación.

Para este caso tomaremos como ejemplo la S/E Pomasqui de la EEQ como se muestra en la Figura 7.

La parte de distribución consta de:

- Un seccionador de barra: 189-B23.

- Siete primarios: 152-A, 152-B, 152-C, 152D, 152-E, 152-F, 152-G.

- Dos Banco de capacitores de 3 MVAR: 152BC1 y 152-BC2. 
Los disyuntores en esta subestación se encuentran en el patio de maniobras como se muestra en la Figura 8.

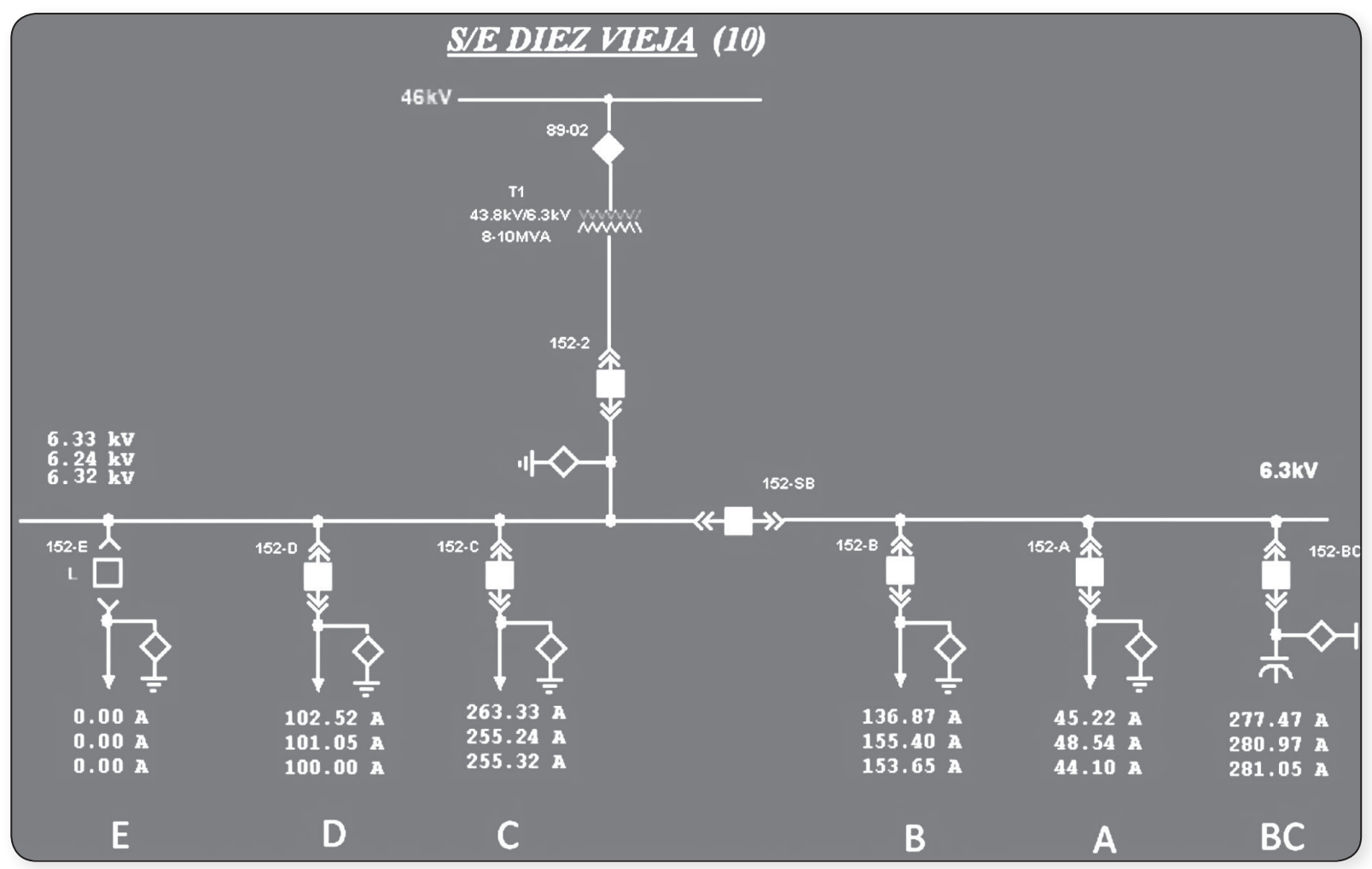

Figura 6. Sinóptico de la S/E Diez Vieja

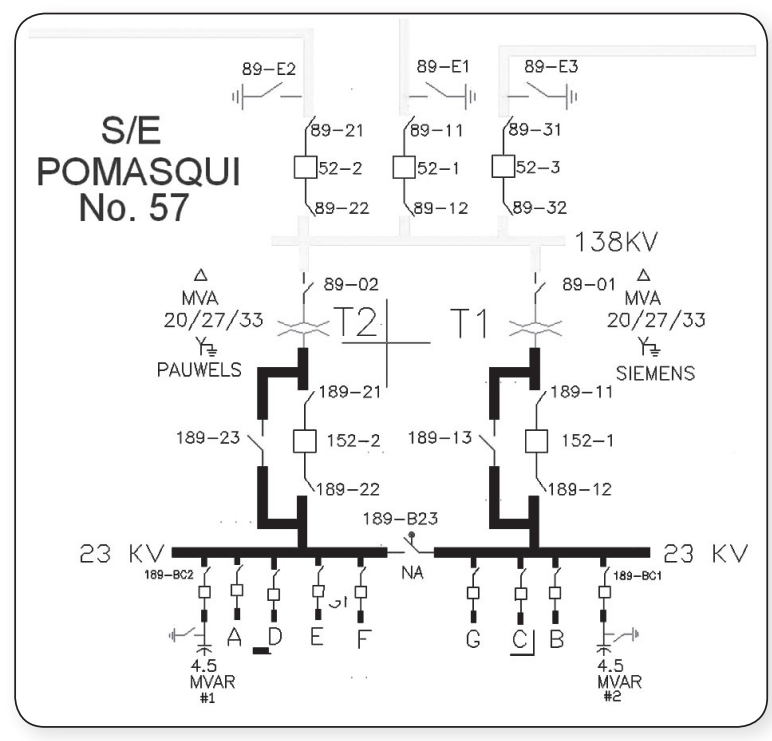

Figura 7. Diagrama unifilar de la S/E Pomasqui

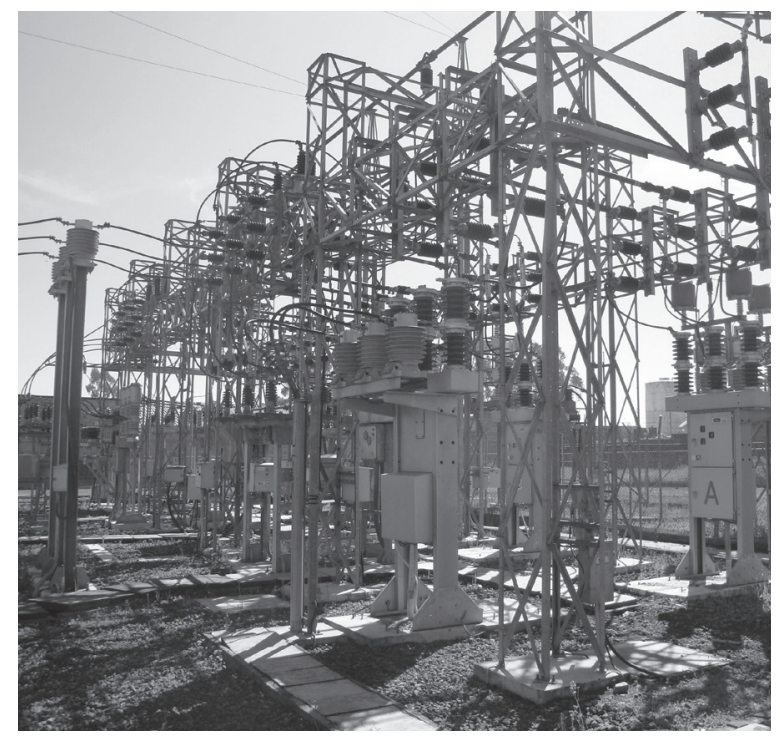

Figura 8. Posición de los disyuntores de $23 \mathrm{kV}$ de la S/E Pomasqui. 
Las Figuras 9 y 10 muestran la vista unifilar superior de la posición de los disyuntores y el esquema de ubicación.

En el sinóptico el orden de dibujo de los disyuntores será para el transformador T2, a partir del seccionador de barra, de izquierda a derecha: BC2, D, A, F y E. Para el T1, será de izquierda a derecha: $\mathrm{G}, \mathrm{BC} 1, \mathrm{~B}$ y $\mathrm{C}$, como se muestra en la Figura 11.

\subsection{Simbología eléctrica utilizada}

En el estándar IEEE/ANSI Std C37.1-1994 [2], se recomienda que: En la interfaz de usuario, para representar datos alfanuméricos, se usarán los caracteres alfanuméricos y sus códigos correspondientes, como los definidos en ANSI INCITS 4-R2002 (ANSI X3.4-1986). Un conjunto mínimo de símbolos gráficos, se recomienda en la Figura 12.

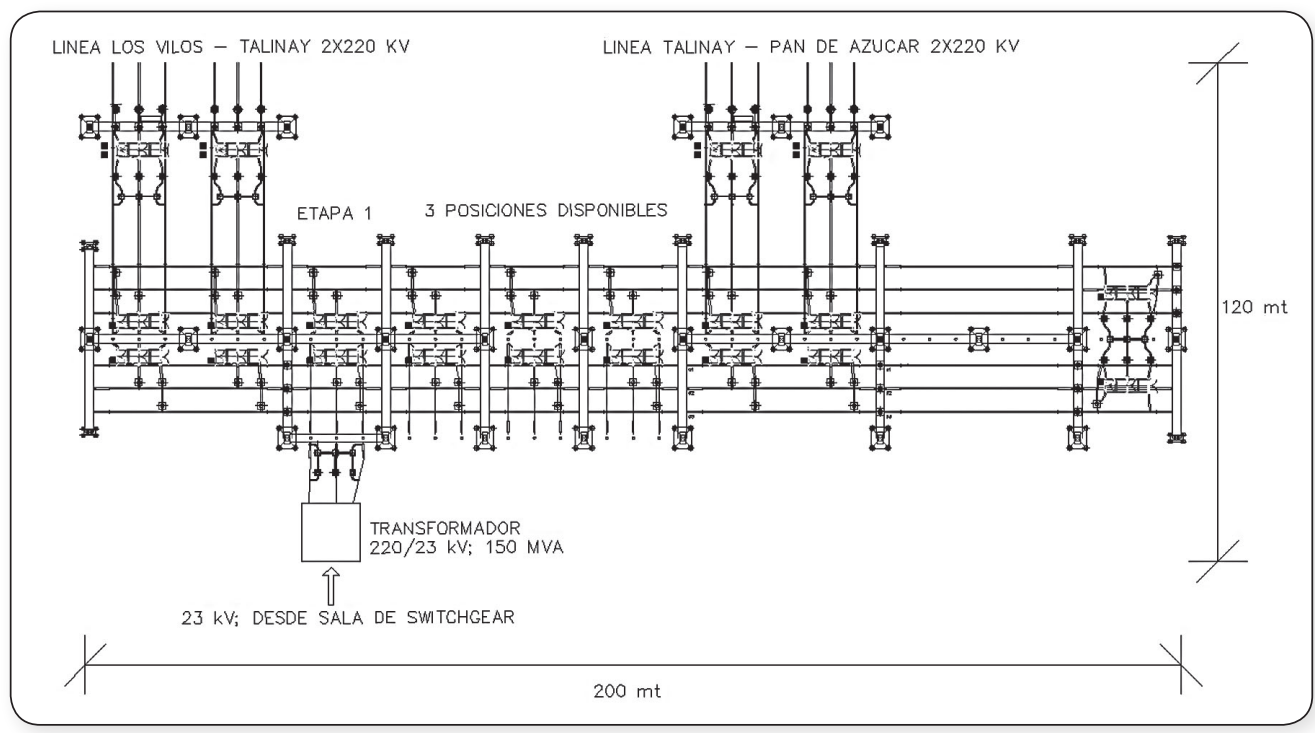

Figura 9. Vista superior de los disyuntores de la S/E Pomasqui

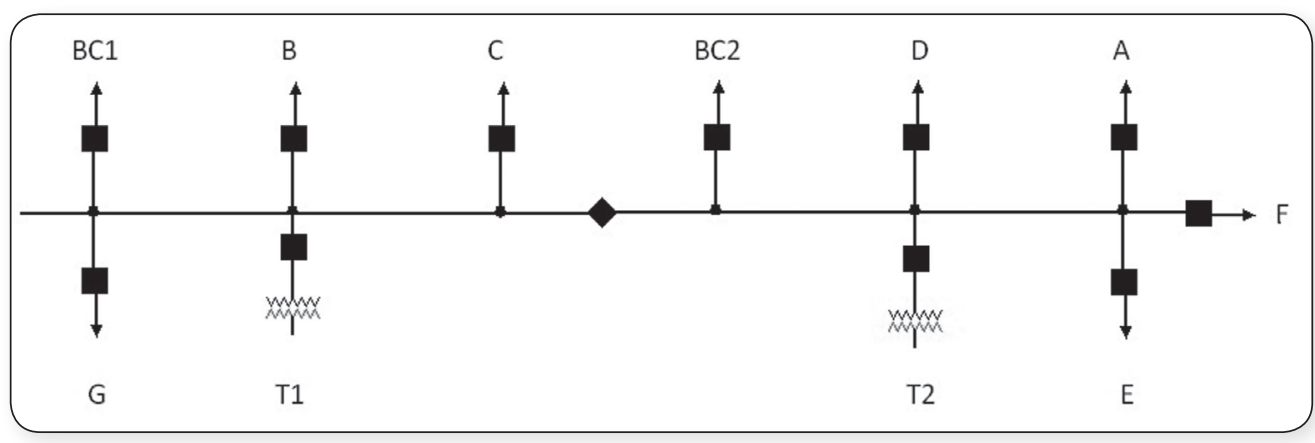

Figura 10. Esquema de ubicación de los disyuntores de la S/E Pomasqui 


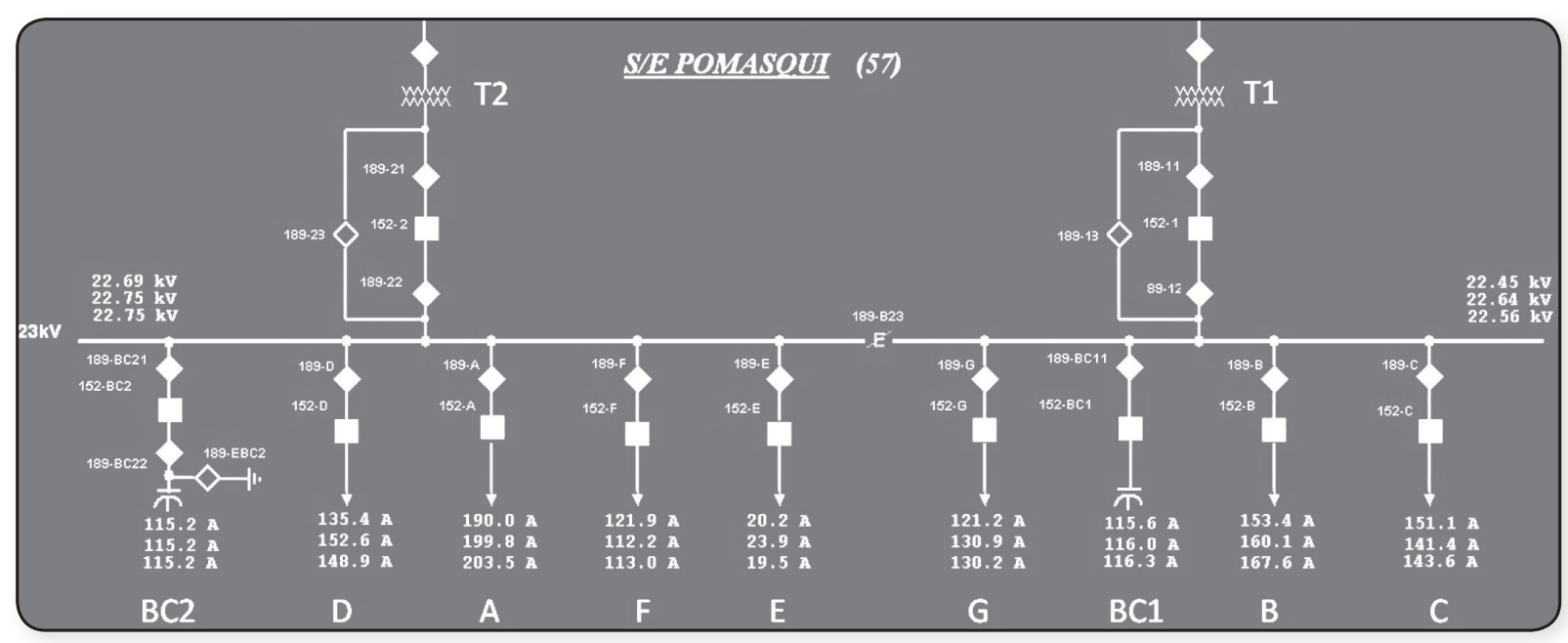

Figura 11. Sinóptico de la S/E Pomasqui

\begin{tabular}{|c|c|c|c|c|c|}
\hline symtol & Maviling & symeol & Moaning & symeol & Mouning \\
\hline & CORNER, UPPER LEFT & & UNE, VERTICAL & & $\begin{array}{l}\text { CORNER, UPPER } \\
\text { REGHT }\end{array}$ \\
\hline & GENERATOR & & UNE, HORIZONTAR & & Bus \\
\hline & OPEN BREAKER & & UNE, CROSSOVER & T & FLOW UP \\
\hline & CLOSED BREAKER & & UNE JUNCTION & $\downarrow$ & FLOW DOWN \\
\hline & OPEN DISCONNECT & & UNE JUNCTION & $\leftarrow$ & FLW LEFT \\
\hline & CLOSEO OISCONNECT & & UNE JUNCTION & $\longrightarrow$ & FOW REST \\
\hline سلد & TRANSFORMER & & UNE JUNCTION & & Bus \\
\hline$\vdash$ & TRANSFORMER & 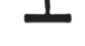 & UNE/BUS JUNCTION & $\uparrow$ & GROUNO SWTTCH \\
\hline$\frac{1}{T}$ & CAPACTIOA & & UNE/BUS JUNCTION & $\rightarrow$ & GAOUND SWTTCH \\
\hline$-1 t$ & CAPACTIOR & & UNE/BUS JUMCTON & $\frac{1}{2}$ & GROUND \\
\hline & CORNER, LOWER LEFT & & UNE/BUS JUNCTION & & $\begin{array}{l}\text { CORNER, LOWER } \\
\text { RIGHT }\end{array}$ \\
\hline
\end{tabular}

Figura 12. Símbolos eléctricos recomendados y significados 
De tal manera que en las especificaciones técnicas para el proyecto Nuevo Sistema SCADA para la EEQ S.A., en el capítulo 4 (Interfaz de Usuario) [4], se recomienda a los oferentes adoptar esta normativa básica de tal forma de uniformizar los criterios de simbología y coloración utilizados en la operación del nuevo sistema SCADA.

La EEQ S.A utiliza los siguientes colores para representar los diferentes niveles de tensión:

\begin{tabular}{|c|c|c|c|c|c|c|}
\hline $\begin{array}{c}\text { Nivel de } \\
\text { Voltaje }\end{array}$ & $230 \mathrm{kV}$ & $138 \mathrm{kV}$ & $46 \mathrm{kV}$ & $23 \mathrm{kV}$ & $13.8 \mathrm{kV}$ & $6.3 \mathrm{kV}$ \\
\hline Color & Azul & Amarillo & Naranja & Verde & Amarillo & Celeste \\
\hline
\end{tabular}

Tabla 1. Color del Nivel de Voltajes

\subsection{Representación dinámica de los elementos de operación}

\section{Disyuntor}

\begin{tabular}{|c|c|c|}
\hline Estado & Representación & Descripción \\
\hline 0 & $\mid 2$ & Disyuntor cerrado \\
\hline 1 & \multicolumn{1}{|c|}{} & Disyuntor abierto \\
\hline 2 & $?$ & Indeterminado \\
\hline 3 & $\mathrm{X}$ & ilegal \\
\hline
\end{tabular}

Carro enganchable

\begin{tabular}{|c|c|c|}
\hline Estado & Representación & Descripción \\
\hline 0 & & $\begin{array}{c}\text { Carro } \\
\text { Desenganchado }\end{array}$ \\
\hline 1 & $\Psi$ & $\begin{array}{c}\text { Carro } \\
\text { Enganchado }\end{array}$ \\
\hline
\end{tabular}

\section{Seccionador}

\begin{tabular}{|c|c|c|}
\hline Estado & Representación & Descripción \\
\hline 0 & & Disyuntor cerrado \\
\hline 1 & & Disyuntor abierto \\
\hline 2 & $?$ & Indeterminado \\
\hline 3 & $\mathrm{X}$ & ilegal \\
\hline
\end{tabular}

\section{Conclusiones y recomendaciones}

En las subestaciones de Distribución, en las cuales se tiene un nivel de tensión de $6,3 \mathrm{kV}$, los disyuntores se encuentran distribuidos en cabinas, dentro o fuera de una sala de control; esto permite tener una primera lógica de ubicación de los equipos; es decir el orden de ubicación de los dispositivos en el sinóptico del SCADA, debe ser el mismo que el de las cabinas. 
En las S/E de Distribución de un nivel de tensión de $23 \mathrm{kV}$, en donde los disyuntores se encuentran en el patio de maniobras, la configuración lógica de los equipos, varia de acuerdo a la ubicación de los disyuntores de alimentación, es decir si se tiene dos disyuntores de alimentación, el seccionador de barra será quien divida hacia un lado y el otro el orden de ubicación de cada disyuntor, de tal forma que en el sinóptico de operación del SCADA, el operador sepa que la disposición del sinóptico es el de la barra y no el de las cabinas de operación.

Para las $S / E$ que tienen los primarios aislados en SF6, la ubicación en el sinóptico será de acuerdo al orden lógico de las cabinas en la sala de control.

En conclusión para las subestaciones en las cuales se tienen cabinas con un nivel de tensión de distribución de $6,3 \mathrm{kV}$ y para las subestaciones en las cuales los disyuntores tienen aislamiento en SF6, la disposición de los equipos en el sinóptico del sistema SCADA indica la ubicación de las cabinas en la sala de control, ya que debido a su diseño y construcción, no se dispone de patio de maniobras, así también para las subestaciones de un nivel de tensión de distribución de $23 \mathrm{Kv}$, la disposición física de los disyuntores indica la distribución de los primaros a nivel del patio de maniobras. |IIII

\section{Referencias bibliográficas}

[1] IEEE Tutorial 94-EH0392-1 PWR. "Fundamentals of Supervisory Systems"

[2] IEEE/ANSI STD C37.1-1994. "IEEE Standard Definition, Specification, and Analysis of Systems Used for Supervisory Control, Data Acquisition, and Automatic Control"

[3] J. Mc. Donald (2003). "Automatización de Subestaciones, SCADA y Comunicaciones”, Seminario Internacional IEEE Power Energy Society, Quito, Ecuador.

[4] J. Orejuela, L. Panchi (2004). "Capítulo 4 Interfaz de Usuario" Especificaciones Técnicos Proyecto Nuevo sistema SCADA EEQ, Quito, Ecuador.

[5] R. Céspedes, V. Orejuela (2006). “Automatización Técnica del Negocio de la Distribución y Protecciones de Redes de Distribución", Congreso Tecnológico de Ibero América IEEE ANDESCON Universidad Politécnica Salesiana, Quito, Ecuador.

[6] L. Panchi (2008). "Proyecto Automatización de Subestaciones, SCADA/DMS y Comunicaciones de la Empresa Eléctrica Quito, S.A.”, Paper Reunión Regional Ramas Estudiantiles y GOLD IEEE, Bogota, Colombia. 